\title{
Cytomorphological diversity of Ageratum Species from North-West India.
}

Arneet Gill and Puja Garg*

Department of Botany, Punjabi University, Patiala 147002, Punjab, India.

Received: February 29, 2016; Revised: March 05, 2016; Accepted: March 18, 2016.

\begin{abstract}
Ageratum belongs to tribe Eupatoriae of family Asteraceae. In the present study floristic forays revealed the prevalence of two species of the genus in North India namely, $A$. conyzoides and $A$. boustonianum. Two morphovariants were observed in both the species. The plants were found to differ on the basis of leaf shape and flower colour. Further, analysis of male meiosis revealed the presence of three cytotypes of $A$. conyzoides and two cytotypes of $A$. houstonianum. The specimens of $A$. conyzoides with purple flowers were found to have chromosome count of $\mathrm{n}=10$ (diploid cytotype, $2 \mathrm{x}$ ) and rarely plants with chromosome count of $2 \mathrm{n}=30$ (triploid cytotype, $3 \mathrm{x}$ ) were also observed. This is the first report of $3 \mathrm{x}$ plants of $A$. conyzoides from wild. The white flowered plants were found to be tetraploid with $\mathrm{n}=20$. In the specimens of $A$. boustonianum, the diploid number $\mathrm{n}=10$ was found in white flowered plants while tetraploid number $\mathrm{n}=20$ was found in purple flowered plants. The meiotic course varies from normal to abnormal in some populations of both the species.
\end{abstract}

Key words: $A$. conyzoides; $A$. boustonianum; Cytotype (2x, 3x and 4x); Morphotype.

\section{Introduction}

Ageratum is one of the plants of family asteraceae that shows wide distribution throughout the World and in India two species, i.e. Ageratum conyzoides and $A$. houstonianum are known to exist (Singh $e$ al., 2011). Ageratum is derived from the greek word 'ageras' which means 'non-aging' and refers to the long lasting nature of flowers. The genus Ageratum includes approximately 30 species of annual and perennial herbs and shrubs that are all native to Central and South America (Okunade, 2002). Belonging to tribe Eupatorieae, the genus is characterised by the absence of yellow corolla and ray florets, presence of tubular corolla with varying shades of blue, lavender or white.

Ageratum conyzoides is also known as Ageratum album Steud., A. caeruleum Hort. ex Poir., $A$. coeruleum Desf., A. cordifolium Roxb., A. birtum Lam., $A$. bumile Salisb., $A$. latifolium Cav., $A$. maritimum H.B. \& K., A. mexicanum Sims., A. obtusifolium Lam., $A$. odoratum Vilm. The plants are malodorous and have peculiar odour like that of goat, hence it is commonly known as Goat weed, Billy goat weed and Chick weed. A. houstonianum is also known as A. mexicanum Sims. and commonly known as Blue billy goat weed, Blue mink, Floss flower, Garden Ageratum and Mexican Ageratum. The species are postulated to have originated in Central America from where they spread as an ornamental to both the Americas and was first introduced in gardens in Europe prior to 1697 from where they became pantropical (Johnson, 1971).

A wide range of bioactive chemical compounds including terpenoids, flavonoids, alkaloids, steroids, chromenes have been recognised from Ageratum species (Kamboj and Saluja, 2008; Adebayo et al., 2011; Singh et al., 2013; Kumar, 2014; Kaur and Dogra, 2014). Ageratum is

\section{Corresponding Author}

Puja Garg

Department of Botany

Punjabi University, Patiala,

Punjab, India. considered an important medicinal plant in folk medicine. It is used for wound dressing, skin diseases, ophthalmia, colic, ulcers, in mouthwash for toothache, killing lice and as anti-itch, antitusive, vermifuge, tonic (Girthens, 1948; Burkill, 1985; Kapur, 1993). The decoction of the plant is used for stomach ailments such as diarrhoea, dysentery, intestinal colic, rheumatism, fever and gynaecological diseases (Sharma and Sharma, 1995; Chopra et al., 2002).

A. conyzoides is distributed throughout India and $A$. houstonianum is known to occur in Northern parts of the country (Sahu, 1982). These plants have naturalised in India. So, the present study has been undertaken to investigate cytomorphological diversity of Ageratum species from North West India.

\section{Materials and Methods}

(a) Area surveyed: Material for male meiotic study was collected on population basis from the different districts of Punjab, Himachal Pradesh, Haryana, Uttarakhand and Jammu \& Kashmir (Table-1).

(b) Morphological studies: The morphology of specimens was studied in situ as well as in vivo. The plants showing variability of apparent morphological features like plant size, leaf shape, size and flower colour were grown in vivo to rule out any differences due to environmental variability between the populations. The comparative study chart was prepared on the basis of in vivo studies. The morphological parameters like plant height, leaf shape, size, flower colour and floral projections were studied for each morphotype to have proper insight of morphological variations. 
(c) Cytological studies: Young flower buds from plants were fixed in carnoy's fixative (Ethanol: Chloroform: Glacial Acetic Acid:: 6:3:1) for 24 hours and then transferred to $70 \%$ alcohol and kept at $4{ }^{\circ} \mathrm{C}$ in the refrigerator until analysis. For male meiotic studies young anthers were smeared in acetocarmine (2\% carmine in $45 \%$ Acetic acid) and number of temporary slides were prepared for each population. Chromosome number was confirmed from several Pollen Mother Cells (PMCs) and microphotographs were taken using Nikon Eclipse $80 i$ microscope fitted with Digital Imaging System.

Apparent pollen fertility will be determined as given by Marks (1954) using glyceroacetocarmine for which the mature anthers from different flowers were squashed in glyceroacetocarmine (Glycerol: acetocarmine :: 1:1) mixture. Well-filled pollen grains with stained nuclei and cytoplasn were scored as fertile while pollen grains that were shrivelled and poorly stained were scored as sterile.
The scanning electron microscopy (SEM) of pollen grains was done in IPLS, DBT Laboratory Punjabi University, Patiala to know the variation in pollen grains at inter and intraspecific levels.

\section{Results}

Cytomorphological studies were conducted on fifty-five populations of Ageratum species from various regions of North West India. The studies revealed prevalence of two species, i.e. A. conyzoides and $A$. houstonianum. The populations of Ageratum showed variation of morphological characters in situ. These were collected and grown in vivo at plant conservatory Punjabi University, Patiala and studied further to substantiate in situ observations. The species were observed to be represented by two morphotypes each (Table-2). Morphotypes of A. conyzoides were found to be different on the basis of colour of corolla and size of the plant, while those of $A$. houstonianum showed variability of corolla colour as well as shape of the leaves (Fig. 1a-t).

Table 1: Information about locality and altitude, accession number, latitude longitude of different types of Ageratum species from different regions of North-West India.

\begin{tabular}{|c|c|c|c|c|}
\hline S.No. & Species & Locality \& Altitude & $\begin{array}{c}\text { Latitude } \\
\text { Longitude }\end{array}$ & $\begin{array}{l}\text { Accession No. } \\
\text { (PUP) }\end{array}$ \\
\hline 1. & \multirow{21}{*}{$\begin{array}{l}\text { A. conyzoides } \\
\text { (Purple, diploid) }\end{array}$} & $\begin{array}{l}\text { Plant Conservatory,Patiala (PB) } \\
(251 \mathrm{~m})\end{array}$ & $30.36^{\circ} \mathrm{N} 76.45^{\circ} \mathrm{E}$ & 59721 \\
\hline 2. & & $\begin{array}{l}\text { Plant Conservatory,Patiala } \quad \text { (PB) } \\
(251 \mathrm{~m})\end{array}$ & $30.36^{\circ} \mathrm{N} 76.45^{\circ} \mathrm{E}$ & 59859 \\
\hline 3. & & Karheri, Patiala (PB) $(251 \mathrm{~m})$ & $30.32^{\circ} \mathrm{N} 76.40^{\circ} \mathrm{E}$ & 59825 \\
\hline 4. & & Ganda Kheri, Patiala (PB) $(251 \mathrm{~m})$ & $30.36^{\circ} \mathrm{N} 76.45^{\circ} \mathrm{E}$ & 59826 \\
\hline 5. & & Sunam, Sangrur (PB) $(231 \mathrm{~m})$ & $30.13^{\circ} \mathrm{N} 75.80^{\circ} \mathrm{E}$ & 59827 \\
\hline 6. & & Sunam Nursery, Sangrur (PB) $(231 \mathrm{~m})$ & $30.13^{\circ} \mathrm{N} 75.80^{\circ} \mathrm{E}$ & 59856 \\
\hline 7. & & Ahmedgarh, Sangrur (PB) $(231 \mathrm{~m})$ & $30.67^{\circ} \mathrm{N} 75.82^{\circ} \mathrm{E}$ & 59857 \\
\hline 8. & & Raikot, Ludhiana (PB) $(254 \mathrm{~m})$ & $30.65^{\circ} \mathrm{N} 75.60^{\circ} \mathrm{E}$ & 59828 \\
\hline 9. & & Ludhiana (PB) $(254 \mathrm{~m})$ & $30.88^{\circ} \mathrm{N} 75.85^{\circ} \mathrm{E}$ & 59855 \\
\hline 10. & & Barnala (PB) $(227 \mathrm{~m})$ & $30.38^{\circ} \mathrm{N} 75.55^{\circ} \mathrm{E}$ & 59833 \\
\hline 11. & & $\operatorname{Moga}(\mathrm{PB})(252 \mathrm{~m})$ & $30.81^{\circ} \mathrm{N} 75.17^{\circ} \mathrm{E}$ & 59832 \\
\hline 12. & & Joga, Mansa (PB) $(217 \mathrm{~m})$ & $30.14^{\circ} \mathrm{N} 75.42^{\circ} \mathrm{E}$ & 59843 \\
\hline 13. & & Hoshiarpur (PB) (293m) & $31.58^{\circ} \mathrm{N} 75.98^{\circ} \mathrm{E}$ & 59831 \\
\hline 14. & & Faridkot $(\mathrm{PB})(196 \mathrm{~m})$ & $30.40^{\circ} \mathrm{N} 75.45^{\circ} \mathrm{E}$ & 59830 \\
\hline 15. & & Kotakpura, Faridkot (PB) (196m) & $30.40^{\circ} \mathrm{N} 75.45^{\circ} \mathrm{E}$ & 59844 \\
\hline 16. & & Sirhind, Fatehgarh Sahib (PB) (246m) & $30.36^{\circ} \mathrm{N} 76.23^{\circ} \mathrm{E}$ & 59852 \\
\hline 17. & & $\operatorname{Ropar}(\mathrm{PB})(260 \mathrm{~m})$ & $31.23^{\circ} \mathrm{N} 76.50^{\circ} \mathrm{E}$ & 59829 \\
\hline 18. & & Anandpur Sahib, Ropar (PB) (260m) & $31.23^{\circ} \mathrm{N} 76.50^{\circ} \mathrm{E}$ & 59862 \\
\hline 19. & & Leisure Valley, Chandigarh $(321 \mathrm{~m})$ & $30.75^{\circ} \mathrm{N} 76.79^{\circ} \mathrm{E}$ & 59835 \\
\hline 20. & & Mohali (PB) $(316 \mathrm{~m})$ & $30.78^{\circ} \mathrm{N} 76.69^{\circ} \mathrm{E}$ & 59860 \\
\hline 21. & & Pathankot (PB) $(335 \mathrm{~m})$ & $32.27^{\circ} \mathrm{N} 75.65^{\circ} \mathrm{E}$ & 59854 \\
\hline 22. & \multirow{19}{*}{$\begin{array}{l}\text { A. conyzoides } \\
\text { (Purple, triploid) }\end{array}$} & Ambala (Haryana) (264) & $30.38^{\circ} \mathrm{N} 76.78^{\circ} \mathrm{E}$ & 59853 \\
\hline 23. & & Panchkula (Haryana) $(365 \mathrm{~m})$ & $30.74^{\circ} \mathrm{N} 76.80^{\circ} \mathrm{E}$ & 59838 \\
\hline 24. & & Una (H.P.) $(369 \mathrm{~m})$ & $31.63^{\circ} \mathrm{N} 76.34^{\circ} \mathrm{E}$ & 59846 \\
\hline 25. & & Bilaspur (H.P.) $(673 \mathrm{~m})$ & $31.33^{\circ} \mathrm{N} 76.75^{\circ} \mathrm{E}$ & 59847 \\
\hline 26. & & Barwana, Bilaspur (H.P.) (673m) & $31.33^{\circ} \mathrm{N} 76.75^{\circ} \mathrm{E}$ & 59848 \\
\hline 27. & & Hamirpur (H.P.) $(785 \mathrm{~m})$ & $31.68^{\circ} \mathrm{N} 76.52^{\circ} \mathrm{E}$ & 59845 \\
\hline 28. & & Sundernagar, Mandi (H.P.) $(1020 \mathrm{~m})$ & $31.53^{\circ} \mathrm{N} 76.90^{\circ} \mathrm{E}$ & 59836 \\
\hline 29. & & Rewalsar, Mandi (H.P.) $(1360 \mathrm{~m})$ & $31.63^{\circ} \mathrm{N} 76.83^{\circ} \mathrm{E}$ & 59849 \\
\hline 30. & & Dharmsala, Kangra (H.P.) (1457m) & $32.21^{\circ} \mathrm{N} 76.32^{\circ} \mathrm{E}$ & 59837 \\
\hline 31. & & Palampur, Kangra (H.P.) (1472m) & $32.11^{\circ} \mathrm{N} 76.53^{\circ} \mathrm{E}$ & 59861 \\
\hline 32. & & Shimla (H.P.) $(2205 \mathrm{~m})$ & $31.10^{\circ} \mathrm{N} 77.17^{\circ} \mathrm{E}$ & 59858 \\
\hline 33. & & Mussorie (Uttarakhand) $(2000 \mathrm{~m})$ & $30.27^{\circ} \mathrm{N} 78.06^{\circ} \mathrm{E}$ & 59834 \\
\hline 34. & & Mussorie (Uttarakhand) (1826m) & $30.45^{\circ} \mathrm{N} 78.06^{\circ} \mathrm{E}$ & 59850 \\
\hline 35. & & Kathua, Jammu (J\&K) (307m) & $32.27^{\circ} \mathrm{N} 76.45^{\circ} \mathrm{E}$ & 59839 \\
\hline 36. & & Kathua, Jammu (J\&K) (307m) & $30.36^{\circ} \mathrm{N} 75.52^{\circ} \mathrm{E}$ & 59851 \\
\hline 37. & & Hamirpur (H.P.) (785m) & $31.68^{\circ} \mathrm{N} 76.52^{\circ} \mathrm{E}$ & 59863 \\
\hline 38. & & Rewalsar, Mandi (H.P.) (1360m) & $31.63^{\circ} \mathrm{N} 76.83^{\circ} \mathrm{E}$ & 59864 \\
\hline 39. & & Rewalsar, Mandi (H.P.) (1360m) & $31.63^{\circ} \mathrm{N} 76.83^{\circ} \mathrm{E}$ & 59865 \\
\hline 40. & & $\begin{array}{l}\text { Plant Conservatory,Patiala (PB) } \\
(251 \mathrm{~m})\end{array}$ & $30.36^{\circ} \mathrm{N} 76.45^{\circ} \mathrm{E}$ & 59722 \\
\hline
\end{tabular}




\begin{tabular}{|c|c|c|c|c|c|}
\hline 41. & & $\begin{array}{l}\text { Plant Conservatory,Patiala } \\
(251 \mathrm{~m})\end{array}$ & (PB) & $30.36^{\circ} \mathrm{N} 76.45^{\circ} \mathrm{E}$ & 59723 \\
\hline 42. & & $\begin{array}{l}\text { Plant Conservatory,Patiala } \\
(251 \mathrm{~m})\end{array}$ & (PB) & $30.36^{\circ} \mathrm{N} 76.45^{\circ} \mathrm{E}$ & 59866 \\
\hline 43. & & $\begin{array}{l}\text { Plant Conservatory,Patiala } \\
(251 \mathrm{~m})\end{array}$ & (PB) & $30.36^{\circ} \mathrm{N} 76.45^{\circ} \mathrm{E}$ & 59867 \\
\hline 44. & & Barsar, Hamirpur (H.P.) (785m) & & $31.68^{\circ} \mathrm{N} 76.52^{\circ} \mathrm{E}$ & 59773 \\
\hline 45. & A. conyzoides & Barsar, Hamirpur (H.P.) (785m) & & $31.68^{\circ} \mathrm{N} 76.52^{\circ} \mathrm{E}$ & 59782 \\
\hline 46. & (White, tetraploid) & Bilaspur (H.P.) (673m) & & $31.33^{\circ} \mathrm{N} 76.75^{\circ} \mathrm{E}$ & 59774 \\
\hline 47. & & Bilaspur (H.P.) (673m) & & $31.33^{\circ} \mathrm{N} 76.75^{\circ} \mathrm{E}$ & 59775 \\
\hline 48. & & Galma, Mandi (H.P.) (1189m) & & $31.62^{\circ} \mathrm{N} 76.87^{\circ} \mathrm{E}$ & 59776 \\
\hline 49. & & Galma, Mandi (H.P.) (1189m) & & $31.62^{\circ} \mathrm{N} 76.87^{\circ} \mathrm{E}$ & 59777 \\
\hline 50. & & Mandli, Una (H.P.) (369m) & & $31.63^{\circ} \mathrm{N} 76.34^{\circ} \mathrm{E}$ & 59778 \\
\hline 51. & & Mandli, Una (H.P.) $(369 \mathrm{~m})$ & & $31.63^{\circ} \mathrm{N} 76.34^{\circ} \mathrm{E}$ & 59779 \\
\hline 52. & & Rewalsar, Mandi (H.P.) (1360m) & & $31.63^{\circ} \mathrm{N} 76.83^{\circ} \mathrm{E}$ & 59780 \\
\hline 53. & & Rewalsar, Mandi (H.P.) (1360m) & & $31.63^{\circ} \mathrm{N} 76.83^{\circ} \mathrm{E}$ & 59781 \\
\hline 54. & $\begin{array}{l}\text { A.houstonianum } \\
\text { (Purple, tetraploid) }\end{array}$ & $\begin{array}{l}\text { Plant Conservatory,Patiala } \\
(251 \mathrm{~m})\end{array}$ & $(\mathrm{PB})$ & $30.36^{\circ} \mathrm{N} 76.45^{\circ} \mathrm{E}$ & 59724 \\
\hline 55. & $\begin{array}{l}\text { A. houstonianum } \\
\text { (White, diploid) }\end{array}$ & $\begin{array}{l}\text { Plant Conservatory,Patiala } \\
(251 \mathrm{~m})\end{array}$ & $(\mathrm{PB})$ & $30.36^{\circ} \mathrm{N} 76.45^{\circ} \mathrm{E}$ & 59725 \\
\hline
\end{tabular}

Table 2: Comparison of cytomorphotypes of Ageratum species from North-West India.

\begin{tabular}{|c|c|c|c|c|c|c|}
\hline \multirow{2}{*}{ S. No. } & \multirow{2}{*}{ Characters } & \multicolumn{3}{|c|}{ Ageratum conyzoides } & \multicolumn{2}{|c|}{ Ageratum houstonianum } \\
\hline & & Purple & Purple & White & Purple & White \\
\hline 1. & Ploidy level & $2 \mathrm{x}$ & $3 \mathrm{x}$ & $4 \mathrm{x}$ & $4 \mathrm{x}$ & $2 \mathrm{x}$ \\
\hline 2. & Habit & Medium sized herb & $\begin{array}{l}\text { Medium sized } \\
\text { herb }\end{array}$ & Small sized herb & Medium sized herb & Small sized herb \\
\hline 3. & Habitat & $\begin{array}{l}\text { Ranging from plains } \\
\text { to high altitude } \\
\text { mountains, along } \\
\text { roads, water bodies } \\
\text { and wheat fields. }\end{array}$ & $\begin{array}{lr}\text { Produced } & \text { in } \\
\text { vivo from } \\
\text { diploid and } \\
\text { tetraploid } \\
\text { plants. }\end{array}$ & $\begin{array}{lr}\text { Along } & \text { with } \\
\text { purple plants in } \\
\text { hilly regions } \\
\text { only. }\end{array}$ & $\begin{array}{l}\text { Escaped } \\
\text { ornamental on low } \\
\text { altitude mountains } \\
\text { and plains usually } \\
\text { under trees. }\end{array}$ & $\begin{array}{l}\text { Escaped } \\
\text { ornamental on low } \\
\text { altitude mountains } \\
\text { and plains usually } \\
\text { under trees. }\end{array}$ \\
\hline 4. & Shape of leaves & $\begin{array}{l}\text { Leaves are } 6.7 \times 5.9 \mathrm{~cm} \\
\text { ovate with obtuse } \\
\text { base never cordate. }\end{array}$ & $\begin{array}{l}\text { Leaves are } \\
5.0 \times 3.1 \mathrm{~cm} \\
\text { ovate with } \\
\text { acute apex. }\end{array}$ & $\begin{array}{l}\text { Leaves are } \\
6.7 \times 3.7 \mathrm{~cm} \\
\text { elliptic-oblong } \\
\text { with acute apex } \\
\text { and base. }\end{array}$ & $\begin{array}{l}\text { Leaves } \\
7.2 \times 6.5 \mathrm{~cm} \\
\text { orbiculate } \\
\text { cordate base. }\end{array}$ & $\begin{array}{l}\text { Leaves are } \\
6.3 \times 5.5 \mathrm{~cm} \quad \text { ovate } \\
\text { with acute apex and } \\
\text { cordate base. }\end{array}$ \\
\hline 5. & $\begin{array}{l}\text { Colour of } \\
\text { inflorescence } \\
\text { Size of }\end{array}$ & Purple & Purple & White & Purple & White \\
\hline 6. & $\begin{array}{l}\text { inflorescence } \\
\text { per capitula }\end{array}$ & $6-7 \mathrm{~mm}$ & $5-6 \mathrm{~mm}$ & $5-6 \mathrm{~mm}$ & 6-8mm & $5-6 \mathrm{~mm}$ \\
\hline 7. & $\begin{array}{l}\text { Involucral } \\
\text { bracts }\end{array}$ & hairy & hairy & less hairy & hairy & less hairy \\
\hline 8. & $\begin{array}{l}\text { Size of floral } \\
\text { projections }\end{array}$ & Large & Large & Small & Large & Small \\
\hline
\end{tabular}

Table 3: Association of chromosomes of triploid plant produced in vivo.

\begin{tabular}{cccc}
\hline \multirow{2}{*}{ No. of PMCs observed } & \multicolumn{4}{c}{ Association of Chromosomes } \\
\cline { 2 - 4 } & I & II & III \\
\hline 4 & 9 & 6 & 3 \\
5 & 7 & 10 & 1 \\
3 & 8 & 11 & - \\
1 & 8 & 8 & 2 \\
2 & 4 & 13 & - \\
3 & 4 & 10 & 2 \\
\hline
\end{tabular}

I: univalent, II: bivalent, III: trivalent.

Table 4: Details of abnormal populations of Ageratum collected from different regions of North-West India.

\begin{tabular}{|c|c|c|c|c|c|c|c|c|c|c|}
\hline \multirow{2}{*}{ S. No. } & \multirow{2}{*}{ Species } & \multirow{2}{*}{ Accession Number PUN } & \multirow{2}{*}{ Ploidy level } & \multicolumn{7}{|c|}{ Abnormalities } \\
\hline & & & & i & ii & iii & iv & $\mathbf{v}$ & vi & vii \\
\hline 1. & & 59825 & $2 \mathrm{x}$ & - & - & - & - & $2.8 \%$ & - & - \\
\hline 2. & & 59826 & $2 \mathrm{x}$ & - & - & $1.9 \%$ & - & $20 \%$ & $4.7 \%$ & - \\
\hline 3. & & 59827 & $2 \mathrm{x}$ & - & - & $2.5 \%$ & - & $11.5 \%$ & - & - \\
\hline 4. & & 59856 & $2 \mathrm{x}$ & & & & & & & \\
\hline 5. & & 59828 & $2 \mathrm{x}$ & $2.5 \%$ & - & - & - & $23.3 \%$ & - & - \\
\hline 6. & A. conyzoides & 59843 & $2 \mathrm{x}$ & $2.2 \%$ & - & - & - & $8.8 \%$ & - & - \\
\hline 7. & (purple) & 59847 & $2 \mathrm{x}$ & - & - & - & $6.6 \%$ & $16.6 \%$ & - & - \\
\hline 8. & & 59848 & $2 \mathrm{x}$ & - & - & - & - & $8.1 \%$ & - & - \\
\hline 9. & & 59845 & $2 \mathrm{x}$ & - & $6.2 \%$ & - & - & - & - & $11.5 \%$ \\
\hline 10. & & 59849 & $2 \mathrm{x}$ & - & - & - & - & - & - & $6.8 \%$ \\
\hline 11. & & 59837 & $2 \mathrm{x}$ & - & - & - & - & - & $8.6 \%$ & - \\
\hline 12. & & 59834 & $2 \mathrm{x}$ & - & - & - & - & $7.9 \%$ & & \\
\hline 13. & A. conyzoides & 59863 & $3 x$ & $2.1 \%$ & $2.3 \%$ & $3.1 \%$ & - & - & - & - \\
\hline
\end{tabular}




\begin{tabular}{|c|c|c|c|c|c|c|c|c|c|c|}
\hline 14. & (purple) & 59864 & $3 \mathrm{x}$ & $1.9 \%$ & $2.9 \%$ & $1.05 \%$ & - & - & - & - \\
\hline 15. & $\begin{array}{l}\text { A. conyzoides } \\
\text { (white) }\end{array}$ & 59778 & $4 \mathrm{x}$ & - & - & - & - & - & $1.7 \%$ & $2.5 \%$ \\
\hline 16. & $\begin{array}{l}\text { A. boustonianum } \\
\text { (purple) }\end{array}$ & 59724 & $4 \mathrm{x}$ & $2.0 \%$ & $3.4 \%$ & $1.3 \%$ & - & $15.5 \%$ & $3.3 \%$ & - \\
\hline
\end{tabular}

i: Vagrant, ii: Laggards, iii: Bridge, iv: Cytomixis, v: Chromosome stickiness, vi: Inter bivalent connection, vii: Early disjunction of bivalent.

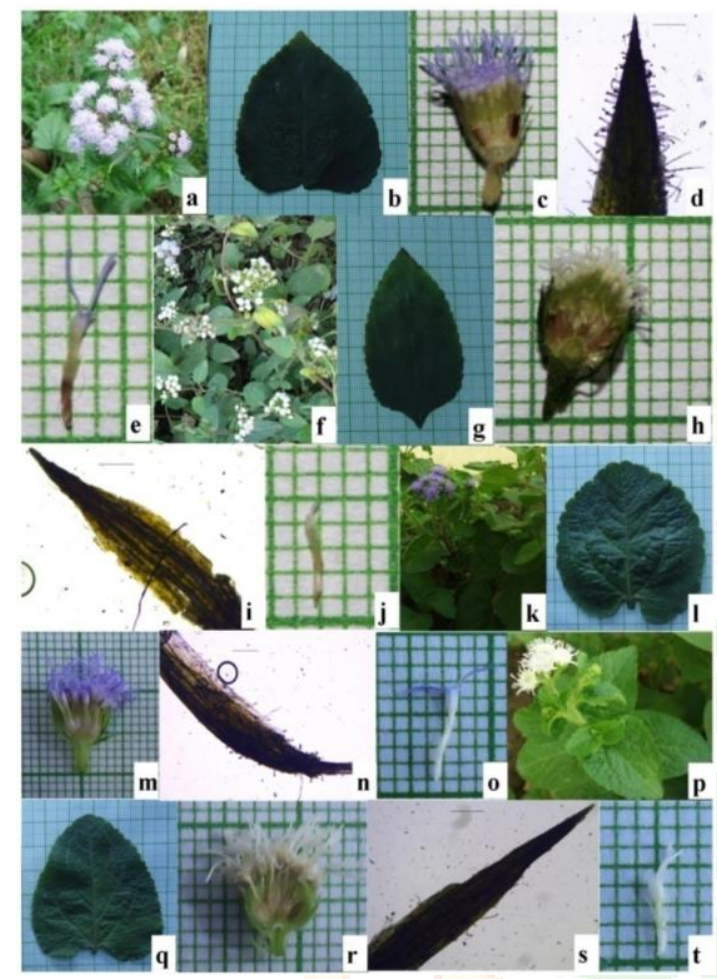

Fig. 1: a) Field photograph of $A$. conyzoides (purple, $2 \mathrm{x})$ b) leaf c) L.S. of capitulum d) involucral bract e) floral pojection f) Field photograph of $A$. conyzoides (white, $4 \mathrm{x}$ ) g) leaf $\mathbf{h}$ ) L.S. of capitulum $\mathbf{i}$ ) involucral bract j) floral projection k) Field photograph of $A$. houstonianum (purple, 4x) 1) leaf m) L.S. of capitulum $\mathbf{n}$ ) involucral bract o) floral projection p) Field photograph of $A$. houstonianum (white, $2 x)$ q) leaf r) L.S. of capitulum s) involucral bract $\mathbf{t}$ ) floral projection.

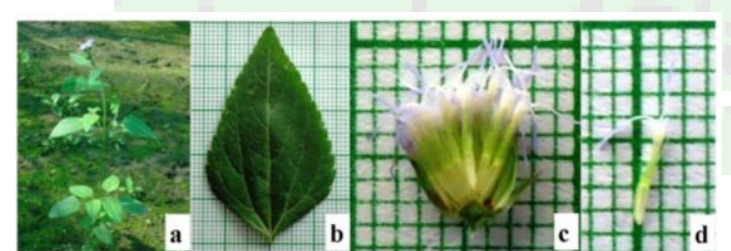

Fig. 2: a) Field photograph of $A$. conyzoides (purple, $3 x)$ b) leaf c) L.S. of capitulum d) floral pojection.

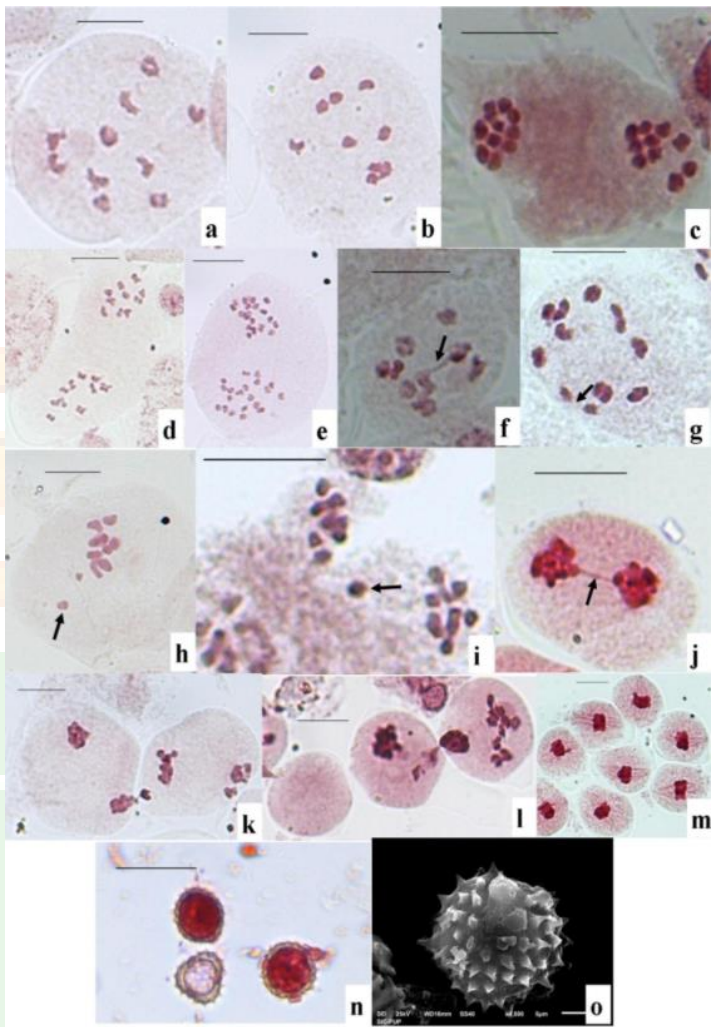

Fig. 3: A. conyzoides $n=10$ a) PMC showing $10 \mathrm{II}$ at Diakinesis b) PMC showing $10 \mathrm{II}$ at M-I c) PMC showing 10:10 chromosomes at A-I d) PMC showing 10:10 chromosomes at M-II e) PMC at mixed A-II f) PMC showing interbivalent connections g) PMC showing early disjunction of bivalents h) PMC showing vagrants i) PMC showing laggards j) PMC showing bridge formation k) PMCs showing cytomixis 1) Hypo-ploid and Hyper-ploid cells m) PMCs showing chromosome stickiness n) Fertile and sterile pollen grains o) Pollen morphology.

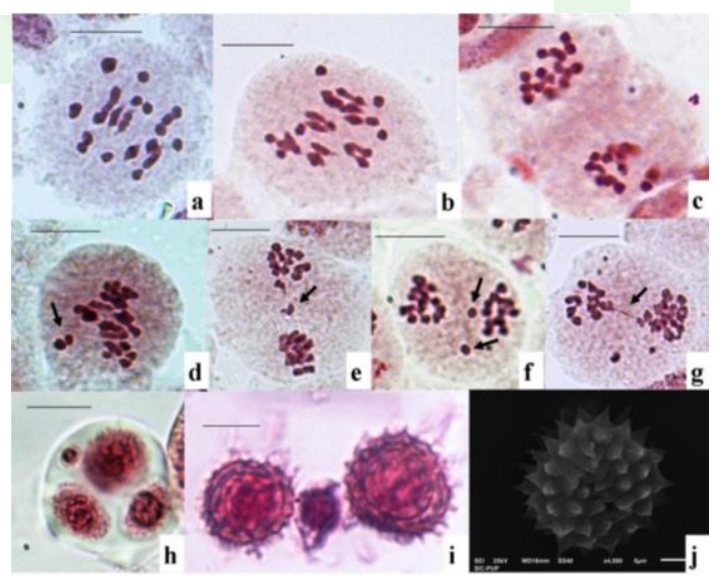

Fig. 4: A. conyzoides $2 \mathrm{n}=30$ a) $\mathrm{PMC}$ showing $3 \mathrm{III}$, $6 \mathrm{II}$ and $9 \mathrm{I}$ at M-I b) PMC showing 1 III, $10 \mathrm{II}$ and 
7 I at M-I c) PMC showing unequal distribution i.e. 16:14 chromosomes at M-II d) PMC showing vagrants e), f) PMC showing laggards g) PMC showing bridge formation $\mathbf{h}$ ) Tetral with micronuclei i) Heterogenous sized fertile pollen grains j) Pollen morphology.

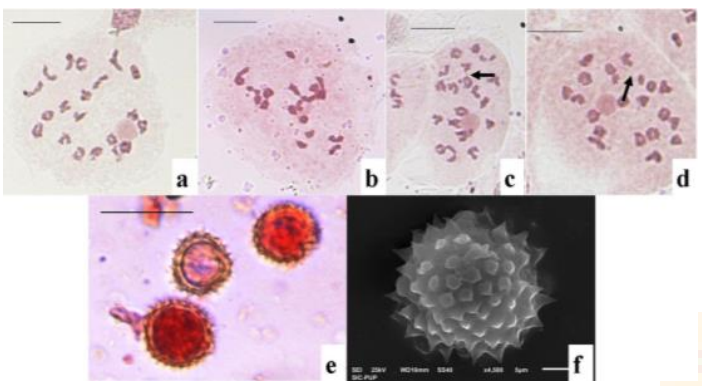

Fig. 5: A. conyzoides $n=20$ a) PMC showing 20 II at Diakinesis b) PMC showing $20 \mathrm{II}$ at M-I c) PMC showing interbivalent connections d) PMC showing early disjunction of bivalents e) Fertile and sterile pollen grains f) Pollen morphology.

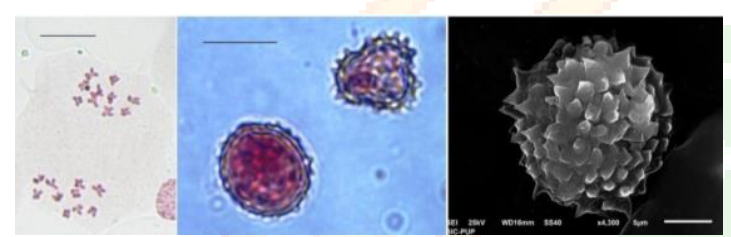

Fig. 6: A. houstonianum $\mathrm{n}=10$ a) $\mathrm{PMC}$ showing 10:10 chromosome distribution at M-II b) Fertile and sterile pollen grains c) Pollen morphology.

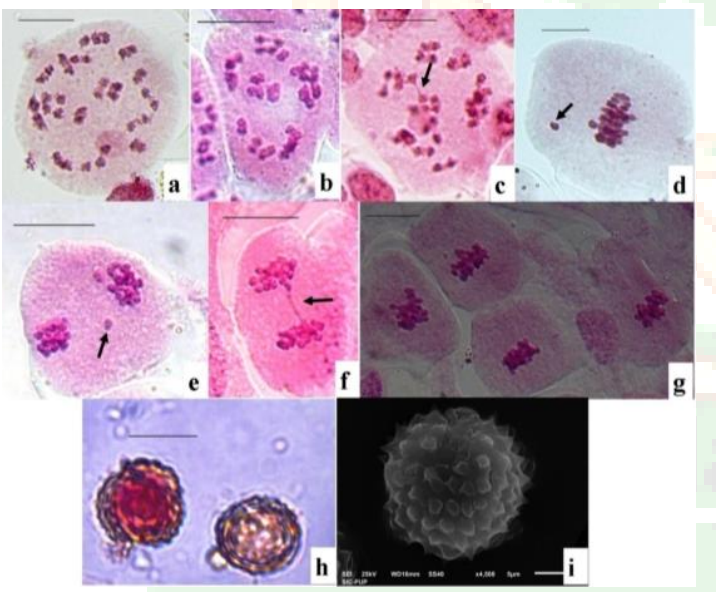

Fig. 7: A. houstonianum $\mathrm{n}=20$ a) $\mathrm{PMC}$ showing 20 $\mathrm{II}$ at Diakinesis b) PMC showing $20 \mathrm{II}$ at M-I c) PMC showing interbivalent connections d) PMC showing vagrants e) PMC showing laggards f) PMC showing bridge formation g) PMCs showing chromosome stickiness h) Fertile and sterile pollen grains iw3) Pollen morphology.

A study of male meiosis showed that the white flowered $A$. conyzoides were tetraploid with $\mathrm{n}=20$ (Fig. 5a-b) while purple flowered $A$. conyzoides were diploid with $\mathrm{n}=10$ (Fig. 3a-e) as well as triploid with $2 \mathrm{n}=30$ (Fig. 4a-c). Out of fifty-three populations of $A$. conyzoides, thirty-six were diploid, four were triploid and thirteen were tetraploid. In the populations of $A$. houstonianum, one was found to be diploid with $\mathrm{n}=10$ (Fig. 6a) and one was tetraploid with $n=20$ (Fig. 7a-b).

The seeds of cytomorphotypes grown in vivo were collected and grown in the subsequent season. The plants that grew from seeds of diploid plants were all diploid while those from tetraploid were also tetraploid in both species. However, some plants growing at the border of $2 \mathrm{n}$ and $4 \mathrm{n}$ populations of A. conyzoides were found to be $3 \mathrm{n}$. This is a first report of in vivo production of triploid $A$. conyzoides plants due to intraspecific hybridization of $2 \mathrm{n}$ and $4 n$ populations. The $3 n$ plants resembled $2 n$ parents morphologically (Fig. 2a-d).

The meiotic course was observed to be abnornal in some populations of Ageratum species. In $A$. conyzoides with $\mathrm{n}=10$ the meiotic abnormalities like interbivalent connections (Fig. 3f), early disjunction of bivalents (Fig. 3g), vagrants (Fig. 3h), laggards (Fig. 3i), bridge formation (Fig. 3j), cytomixis (Fig. 3k), hyper-ploid and hypoploid cells (Fig. 31), chromosome stickiness (Fig. 3m) were observed in the populations collected from Karheri, Ganda kheri, Sunam, Sunam nursery, Raikot, Mansa, Bilaspur, Barwana, Hamirpur, Rewalsar, Dharmsala and Mussorie (Table-4). These spindle abnormalities resulted into heterogenous sized fertile and sterile pollen grains (Fig. 3n). A detailed study of meiotic course revealed that triploids showed secondary association of chromosomes (Table-3) and meiotic irregularities like secondary associations vagrants (Fig. 4d), laggards (Fig. 4e-f), bridges (Fig. 4g) and abnormal microsporogenesis (Fig. 4h) were also observed. The white plants of $A$. conyzoides collected from Una district showed some meiotic abnormalities like interbivalent connections (Fig. 5c) and early disjunction of bivalents (Fig. 5d). The meiotic course was observed to be abnormal in purple plants of $A$. houstonianum with some meiotic irregularities like interbivalent connections (Fig. 7c), vagrant (Fig. 7d), laggard (Fig. 7e), bridge formation (Fig. 7f) and chromosome stickiness (Fig. 7g). Meiotic abnormalities in the species may lead to some pollen sterility and heterogenous sized pollen grains (Fig. 7h).

The scanning electron microscopic studies of pollens of Ageratum revealed that pollen grains are rounded in shape, tritreme and trichotomocolpate. Exine was observed to be echinate with similar morphology and size in all the morphotypes (Fig. 3n-o, 4i-j, 5e-f, 6b-c, 7h-i).

\section{Discussion}

In $A$. conyzoides the meiotic chromosome number of $2 \mathrm{n}=20$ (Fig. 3a-e) was in line with the previous records by large number of workers including Ishikawa (1916), Mehra and Sidhu (1960), Kaul 
(1967), Bir and Sidhu (1979), Sidhu and Pelia (1987), Gupta and Gill (1989), Gaonkar and Torne (1991). Earlier Nazeer et al., (1981) have reported the triploid cytotype as interspecific hybrid of $A$. conyzoides and $A$. boustonianum. The chromosome number of $2 n=40$ agrees with the earlier records of Love and Love (1961), Turner and King (1964), Kaul (1971), Subramanyam and Kamble (1971), Mehra and Remanandan (1975), Keil et al., (1988), Nirmala and Rao (1989) and Xie and Zheng (2003). Earlier Gill and Gupta (1971) reported the presence of $0-3 \mathrm{~B}$ chromosomes in PMCs of Ageratum conyzoides. Dey (1979) reported the diploidy and tetraploidy in $A$. conyzoides. The species is also known to have chromosome counts of $2 \mathrm{n}=36$ (Husaini and Iwo, 1990), $2 \mathrm{n}=38$ (Sharma and Verma, 1960; Chen et al., 2003). Singhal et al., (2013) recorded the early disjunction of bivalents in diploid cytotype from Mandi district of Himachal Pradesh. Meiotic abnormalities in Ageratum conyzoides from Rajasthan was reported by Ramanpreet and Gupta (2015).

In the plants of $A$. houstonianum, the diploid cytotype with $2 \mathrm{n}=20$ in white flowered plants was in line with the previous records by Copper and Mahony (1935), Hsu (1967, 1970), Sharma and Dhakre (1981). The chromosome counts of $2 \mathrm{n}=40$ was reported previously by Shukur et al., (1977).

The cytological studies in both the species of Ageratum was done by Mehra et al., (1965), Miyagi (1971), Mathew and Mathew (1983, 1988), George et al., (1989), Morton (1993) and Razaq et al., (1994).

\section{Conclusion}

The present study showed prevalance of two species of Ageratum in the study area of which $A$. conyzoides was found growing luxuriously in wild howerver $A$. houstonianum was collected only from cultivation. There are two cytomorphotypes of $A$. boustonianum and three of $A$. conyzoides prevalant in the area. The present investigations put forward a report of $3 \mathrm{x}$ intraspecific hybrid (2x $\mathrm{X} 4 \mathrm{x})$ of $A$. conyzoides for first time.

\section{Acknowledgement}

The authors acknowledge the financial and material facilities provided by University Grants Commission, New Delhi under DRS and BSR programmes. Thanks are due to the Head, Department of Botany and DBT IPLS project, Punjabi University, Patiala for providing necessary infrastructural facilities.

\section{References}

1. Adebayo AH, CJ Ji, YM Zhang, WJ He, GZ Zeng, HJ Han, JJ Xu, AA Akindahunsi, NH Tan. "A new chromene isolated from Ageratum conyzoides" Natural Product Communications 6.9 (2011): 1263-1265.
2. Bir SS, M Sidhu. "Cytological observations in weed flora of orchards of Patiala district, Punjab". Recent Research in Plant Sciences 7 (1979): 261-271.

3. Burkill HM. "The useful plants of West Tropical Africa, Kew: Royal Botanic Garden:”. 1 (1985).

4. Chen RY, WQ Song, XL Li, MX Li, GL Liang, CB Chen. "Chromosome atlas of major economic plants genome in China”. Chromosome Atlas of Garden Flowering Plants in China Science Press, Beijing 3 (2003).

5. Chopra RN, SL Nayar, IC Chopra. "Glossary of Indian Medicinal Plants". New Delhi NISCIR (2002): 9.

6. Cooper DC, KL Mahony. "Cytological observations on certain compositae". American Journal of Botany 22 (1935): 843-849.

7. Dey D. "Diploidy and tetraploidy in Ageratum conyroides L". Cell and chromosome Newsletter 2 (1979): 7 9.

8. Gaonkar RV, SG Torne. "Induced autotetraploidy in Ageratum conyzoides L”. Cytologia 56 (1991): 327-331.

9. George S, V Mathew, PM Mathew. "Cytology of a few south Indian Eupatorieae (Compositae)". Glimpses Cytogenetics 2 (1989): 293-298.

10. Gill BS, T Gupta. "B- chromosomes in Ageratum conyroides". Proceedings of $58^{\text {th }}$ Indian Science Congress 3 (1971): 477-478.

11. Girthens TS. "Drug plants of Africa". African Handbooks 8 (1948): 59.

12. Gupta RC, BS Gill. "Cytopalynology of North and Central Indian Compositae". Journal of Cytology and Genetics 24 (1989): 96-105.

13. Hsu CC. "Preliminary chromosome studies on the vascular plants of Taiwan (I)". Taiwania 13 (1967): 117-130.

14. Hsu CC. "Preliminary chromosome studies on the vascular plants of Taiwan (III) The aster family, Compositae”. Taiwania 15 (1970): 17-29.

15. Husaini SWH, GA Iwo. "Cytology of some weedy species of the family Compositae (Asteraceae) from Jos Plateau Nigeria". Feddes Repertorium 101 (1990): 49 62.

16. Ishikawa M. "The chromosome numbers of some Compositae". Botanical Magazine 25 (1916): 399.

17. Johnson MF. "A monograph of the genus Ageratum L. (Compositae, Eupatorieae)". Annals of the Missouri Botanical Garden 58 (1971): 6-88.

18. Kamboj A, AK Saluja. "Ageratum conyzoides L.: A review on its phytochemical and pharmacological profile". International Journal of Green Pharmacy 2 (2008): 59-68.

19. Kapur SK. "Ethnomedico plants of Kangra Valley (Himachal Pradesh)". Journal of Economic and Taxonomic Botany 17 (1993): 395-408. 
20. Kaul MLH. "Cytogenetics of polyploids. III. Dauermodifications in Ageratum conyzoides L". Cytologia 32 (1967): 147-156.

21. Kaur R, NK Dogra. "A review on traditional uses, chemical constituents and pharmacology of Ageratum conyzoides L. (Asteraceae)". International Journal of Pharmaceutical and Biological Archives 5.5 (2014): 33-45.

22. Keil DJ, MA Luckow, DJ Pinkava. "Chromosome studies in Asteraceae from the United States, Mexico, the West Indies, and South America". American Journal of Botany 75 (1988): 652-668.

23. Kumar N. "Biological potential of a weed Ageratum houstonianum Mill: A Review". Indo American Journal of Pharmaceutical Research 4.6 (2014): 2683-2689.

24. Love A, D Love. "Chromosome numbers of central and north west European plant species". Opera Botanica 5 (1961): 1-581.

25. Marks GE. "An acetocarmine glycerol jelly for use in pollen fertility counts”. Stain Techniques 29 (1954): 277.

26. Mathew A, PM Mathew. "Cytological studies on the south Indian Compositae". Glimpses of Plant Research 8 (1988): 1-177.

27. Mathew PM, A Mathew. "Studies on the south Indian Compositae V. Cytotaxonomic consideration of the tribes Vernonieae and Eupatorieae". Cytologia 48 (1983): 679-690.

28. Mehra PN, P Remanandan. "Cytological investigations on Indian Compositae IV. Tribes Senecioneae, Eupatorieae, Vernonieae and Inuleae". Nucleus 18 (1975): 6-19.

29. Mehra PN, SS Sidhu. "Cytology of the North-West Indian Compositae". Proceedings of $47^{\text {th }}$ Indian Science Congress 3 (1960): 376-377.

30. Mehra PN, BS Gill, JK Mehta, SS Sidhu. "Cytological investigations on the Indian compositae I North Indian taxa". Caryologia 18 (1965): 35-68.

31. Miyagi C. "Chromosome numbers in spermatophytes from Okinawa Islands (1)". Biological Magazine Okinawa 8 (1971): 14-23

32. Morton JK. "Chromosome numbers and polyploidy in the flora of Cameroon Mountain". Opera Botanica 121 (1993): 159-172.

33. Nazeer MA, GV Subramanyam, KJ Madusoodanan, D Ohri. "Cytology of triploid hybrid of Ageratum Linn”. Current Science 50 (1981): 97-98.

34. Nirmala A, PN Rao. "Karyotype studies in some Asteraceae”. Cell Chromosome Research 12 (1989): 17-18.

35. Okunade AL. "Ageratum conyzoides L. (Asteraceae)". Fitoterapia 73 (2002): 1-16.
36. Ramanpreet, RC Gupta. "Meiotic abnormalities in Ageratum conyzoides from hot desert of India (Rajasthan)". Chromosome Botany 10 (2015): 67-74.

37. Razaq ZA, AA Vahidy, SI Ali. "Chromosome number in Compositae from Pakistan". Annals of Missouri Botanical Garden 81 (1994): 800-808.

38. Sahu TR. "Taxonomic studies of the genus Ageratum L. in India”. Feddes Repertorium 93 (1982): 61-65.

39. Sharma AK, JS Dhakre. "In chromosome number reports LXXIII”. Taxon 30 (1981): 854.

40. Sharma AK, B Verma. "Cytology of several members of Compositae I". Proceedings of 47th Indian Science Congress 3 (1960): 367.

41. Sharma PD, OP Sharma. "Natural products chemistry and biological properties of the Ageratum plant". Toxicology Environmental Chemistry 50 (1995): 213.

42. Shukur A, KN Narayan, C Shantamma. "In IOPB Chromosome number reports LV". Taxon 26 (1977): 107-109.

43. Sidhu M, SS Pelia. "Karyomorphology of some species of weeds - Compositae". Journal of Cytology and Genetics 22 (1987): 143-150.

44. Singh SB, WR Devi, A Marina, WI Devi, N Swapana, $\mathrm{CB}$ Singh. "Ethnobotany, phytochemistry and pharmacology of Ageratum conyzoides Linn (Asteraceae)". Journal of Medicinal Plants Research 7.8 (2013): 371-385.

45. Singh V, H Singh, GP Sharma, AS Raghubanshi "Eco-physiological performance of two invasive weed congeners (Ageratum conyzoides L. and Ageratum houstonianum Mill.) in the Indo-Gangetic plains of India". Environmental Monitoring and Assessment 178 (2011): 415-422.

46. Singhal VK, P Garg, P Kumar. "Cytological studies of some Dicots from the Hills of Mandi district (Himachal Pradesh) in Northwest Indian Himalayas". Cytologia 78.1 (2013): 55-68.

47. Subramanyam K, NP Kamble. "In: IOPB Chromosome number reports XII". Taxon 16 (1967): 341-350.

48. Turner BL, RM King. "Chromosome numbers in the Compositae VIII. Mexican and Central American species". South Western Naturalist 9 (1964): 27-39.

49. Xie ZY, CM Zheng. "Cytological studies on 13 species of Compositae from Hainan, China". Acta Phytotaxonomica Sinica 41.6 (2003): 545-552.

\section{Cite this article as:}

Arneet Gill and Puja Garg. Cytomorphological diversity of Ageratum Species from North-West India. Annals of Plant Sciences 5.3 (2016): 1291-1295. 